\title{
Role of Electron Illumination on the Image Resolution and Sensitivity of Catalysts Immersed in Gas Environments
}

S. P. Jespersen ${ }^{1,2}$, M. Ek ${ }^{1,3}$, J. R. Jinschek ${ }^{4}$, B. Hendriksen ${ }^{5}$, L. Mele ${ }^{5}$, P. Dona ${ }^{5}$, C. D. Damsgaard ${ }^{2}$ and S. Helveg ${ }^{1}$

1. Haldor Topsoe A/S, Lyngby, Denmark.

2. Department of Physics and Center for Electron Microscopy, Technical University of Denmark, Lyngby, Denmark.

3. Centre for Analysis and Synthesis, Lund University, Lund, Sweden.

4. Department of Material Science and Engineering, Ohio State University, USA.

5. Thermo Fisher Scientific, Eindhoven, The Netherlands.

Progress in gas cell technology has made transmission electron microscopy (TEM) capable of imaging heterogeneous catalysts in situ during exposure to reactive gas environments [1]. While these instrumental advances have opened up for impressive TEM studies of catalyst dynamics at the atomicscale, it has also become evident that the electron beam can substantially alter the catalyst and gas phase and, in turn, the chemical processes under inspection. It therefore becomes crucial to address the impact of electron illumination on in situ TEM observations and to suppress the electron-induced alterations to enable chemical relevant observations [2-4].

Here, we address the role of electron illumination on TEM observations of heterogeneous catalysts immersed in reactive gas environments by measuring the achievable image resolution and sensitivity under a systematic variation of the electron dose-rate, total dose and energy. The measurements were done using the most common types of gas cells including a differentially pumped electron microscopy (FEI Titan 80-300 ETEM) operated at gas pressures of up to 20 mbar [2-3], and a micro-electromechanical-system-based nanoreactor (Thermo Fisher Scientific), operated at gas pressures up to 1 bar [5-6]. The image sensitivity was defined as the signal-to-noise ratio detected by the CCD camera and the image resolution was measured as the shortest transferred lattice vector for a $\mathrm{Au} / \mathrm{C}$ sample in the differentially pumped gas cell and for Pt nanocrystals in the nanoreactor (Figure 1) $[2,4]$.

For the differentially pumped microscope, the image sensitivity drops approximately exponentially with increasing pressure and exhibits a dose-dependency [2]. In contrast, the image resolution has a marked dependency on the dose-rate for a fixed dose (Figure 1). Specifically, the resolution degrades for high dose-rates in the presence of gas while resolution is maintained under high vacuum conditions. Interestingly, Figure 1 shows that the inherent $1 \AA$ resolution of the host microscope can be maintained as long as the electron dose-rate is kept sufficiently low [2-4]. Detailed measurements of the image resolution as a function of time, sample temperature and gas pressure suggest that the electron dose-ratedependent resolution is caused by a sample charging, induced by the beam-gas interaction, that eventually leads to sample motion and hence contrast smearing [4]. For the nanoreactor, similar measurements indicate that the inherent microscope resolution can be preserved even for gas atmospheres at the 1 bar level [5-6].

The present findings that the image resolution can be maintained at electron dose-rates of roughly $100 \mathrm{e}^{-}$ $\AA^{-2} \mathrm{~s}^{-1}$ suggest, in general, that low dose-rates are crucial for suppressing beam-induced sample 
alterations. In practice, however, a low dose-rate results in a vanishing image signal at high magnifications so averaging over series of consecutively acquired images, e.g. by means of in-line holography, is needed to recover a detectable signal $[3,4]$. This concept of low electron dose-rates should readily benefit complementary TEM techniques used for in situ observations of catalysts.

References:

[1] S. Helveg, J. Catal. 328 (2015), p. 102.

[2] J. R. Jinschek \& S. Helveg, Micron 43 (2012), p. 1156.

[3] S. Helveg et al, Micron 68 (2014), p. 176.

[4] M. Ek, et al, Advanced Structural and Chemical Imaging 2 (2017), p. 4.

[5] J. F. Creemer et al, Ultramicroscopy 108 (2008), p 993.

[6] S.B. Vendelbo et al, Nature Materials 13 (2014), p. 884.

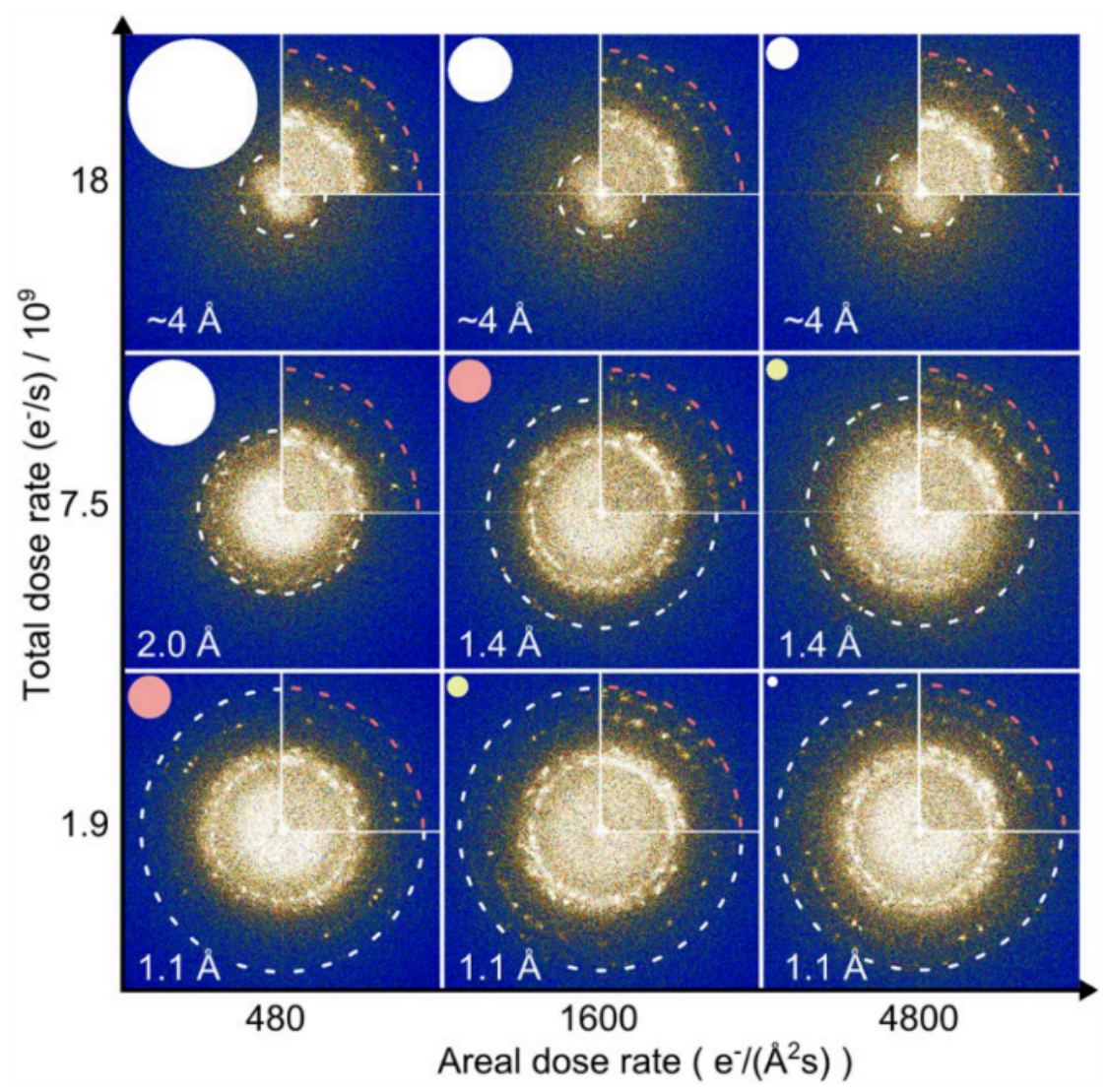

Figure 1. Image resolution as a function of total and areal electron dose-rate. The display are FFTs of high-resolution TEM images of a $\mathrm{Au} / \mathrm{C}$ sample under exposure on 5 mbar $\mathrm{N}_{2}$. Inserts correspond to a FFT of an image acquired under high vacuum conditions $\left(\sim 1 \times 10^{-6} \mathrm{mbar}\right)$. All images were acquired with a single exposure of the CCD for $0.25 \mathrm{~s}$ to $6 \mathrm{~s}$ in order to keep a constant total electron dose for all images. The relative beam-sizes used to maintain a specific areal dose-rate are indicated by the full colored circles superimposed on the FFTs. Figure adopted from [4]. 